\title{
A new technique to measure micromotion distribution around a cementless femoral stem
}

\author{
Michael Gortchacow ${ }^{\mathrm{a}}$, Michael Wettstein ${ }^{\mathrm{b}}$, Dominique P. Pioletti ${ }^{\mathrm{a}}$, Alexandre Terrier ${ }^{\mathrm{a}, *}$ \\ a Laboratory of Biomechanical Orthopedics, Ecole Polytechnique Fédérale de Lausanne, Station 15, 1015 Lausanne, Switzerland \\ b Service of Orthopaedics and Traumatology, University Hospital Center and University of Lausanne, Rue du Bugnon 46, 1011 Lausanne, Switzerland
}

\section{A R T I C L E I N F O}

\section{Article history:}

Accepted 17 September 2010

\section{Keywords:}

Micromotions

Arthroplasty

Hip

MicroCT

\begin{abstract}
A B S T R A C T
The interfacial micromotion is closely associated to the long-term success of cementless hip prostheses. Various techniques have been proposed to measure them, but only a few number of points over the stem surface can be measured simultaneously. In this paper, we propose a new technique based on micro-Computer Tomography $(\mu \mathrm{CT})$ to measure locally the relative interfacial micromotions between the metallic stem and the surrounding femoral bone. Tantalum beads were stuck at the stem surface and spread at the endosteal surface. Relative micromotions between the stem and the endosteal bone surfaces were measured at different loading amplitudes. The estimated error was $10 \mu \mathrm{m}$ and the maximal micromotion was $60 \mu \mathrm{m}$, in the loading direction, at $1400 \mathrm{~N}$. This pilot study provided a local measurement of the micromotions in the 3 direction and at 8 locations on the stem surface simultaneously. This technique could be easily extended to higher loads and a much larger number of points, covering the entire stem surface and providing a quasi-continuous distribution of the 3D interfacial micromotions around the stem. The new measurement method would be very useful to compare the induced micromotions of different stem designs and to optimize the primary stability of cementless total hip arthroplasty.
\end{abstract}

(c) 2010 Elsevier Ltd. All rights reserved.

\section{Introduction}

Primary stability of femoral stems is a crucial condition to guarantee the long-term success of cementless total hip arthroplasty (THA). Animal experiments have indeed reported bone ingrowth for micromotion below $28 \mu \mathrm{m}$ and connective tissue formation above $150 \mu \mathrm{m}$ (Pilliar et al., 1986; Jasty et al., 1997). This was also observed on 12 THA patients at autopsy, who revealed failed bone ingrowth for $150 \mu \mathrm{m}$ of micromotion and bone ingrowth for $40 \mu \mathrm{m}$ of micromotion (Engh et al., 1992).

The effort of the last 20 years in developing techniques for measuring micromotion attests its importance to improve the survival of cementless total hip replacement. However, even if the problem is clearly observed and partly quantified, measurement methods are not fully satisfactory. Most of the studies are using linear variable differential transducers (LVDT's) (Walker et al., 1987; Gilbert et al., 1992; Buhler et al., 1997; Britton et al., 2004; Gheduzzi and Miles, 2007) which do not really measure the local relative micromotion between the stem and the bone, but also include bone deformation between the device fixation and the measurement site. Besides, the number of simultaneous

\footnotetext{
* Corresponding author. Tel.: +4121693 9994; fax: +41216938660.

E-mail address: alexandre.terrier@epfl.ch (A. Terrier).
}

measurement points is limited to 1 or 2 , with a reported maximum of 4 (Cristofolini et al., 2003).

Therefore, the goal of this pilot study was to assess the feasibility of a new technique using $\mu \mathrm{CT}$ to measure the local interfacial micromotions around a femoral stem at a large number of locations simultaneously. To obtain a proof-of-concept of this technique, we tested the method with 8 simultaneous locations.

\section{Methods}

A cementless femoral stem (SPS, Symbios Orthopédie SA, Switzerland) made of titanium alloy $\left(\mathrm{Ti}_{6} \mathrm{Al}_{4} \mathrm{~V}\right.$ ) was inserted into a cadaveric femur (Anatomy Department, University Hospital of Lausanne) by a senior surgeon according to manufacturer recommendations. Bone resection and reaming were performed as in the operation room. Prior to implantation, 5 spherical $(\emptyset 800 \mu \mathrm{m})$ tantalum $(\mathrm{Ta})$ beads (stem beads) were super-glued in drilled holes on the stem surface. These beads were positioned so as to form approximately a dipyramid in order to determine the rigid motion of the stem. Other 15 Ta beads (bone beads) were manually press fit at different locations of the reamed peri-implant bone surface, to measure the micromotion at these locations.

The epiphysis was removed by performing a cut perpendicular to the femoral axis. The distal end was then cemented (Beracryl, Troller AG, Switzerland) within an aluminum cylindrical support. This support was then fixed with 6 (radial) screws to a cylindrical loading device enclosing the femur (Fig. 1). The loading was applied by a piston sliding within the cylinder, and controlled manually in displacement. Coned-spacer springs were used to avoid peaks of load during the manual loading process. The neck of the stem was cut to apply the piston 

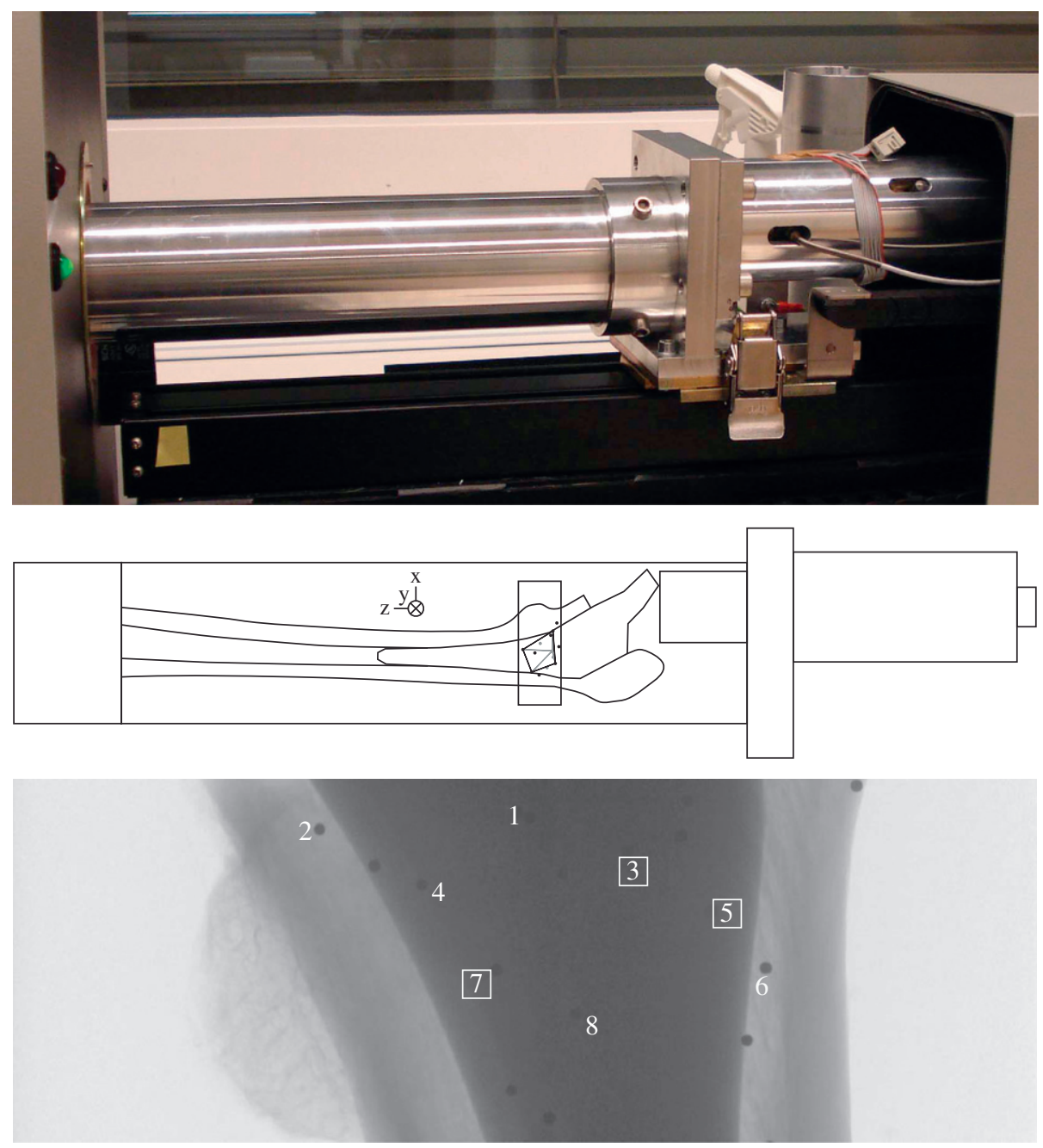

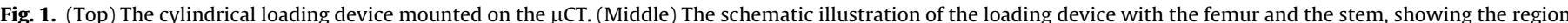

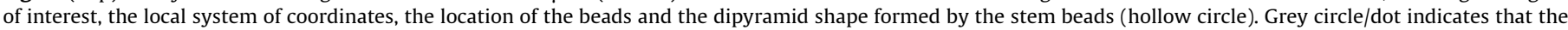

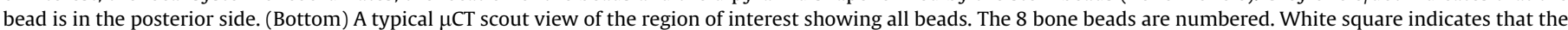
bead is in the posterior side.

compressive force at the rotation center of the femoral head. The cylindrical loading device enclosing the femur was specifically designed to operate inside a $\mu \mathrm{CT}$ (1076 in vivo microCT, Skyscan, Belgium).

A compressive load was applied in 7 successive steps: $0,300,600,900,1200$ 1400 and back to $0 \mathrm{~N}$. Incremental loading was chosen to estimate the effect of load on error, the loading associated to $150 \mu \mathrm{m}$ of micromotion, and the subsidence. The $\mu \mathrm{CT}$ parameters were optimized $(U: 100 \mathrm{kV}, I: 100 \mu \mathrm{A}$, exposure: $240 \mathrm{~ms}$, resolution: $36 \mu \mathrm{m}$, rotational step: $\left.1^{\circ}\right)$. The measurement time of a step was approximately $40 \mathrm{~min}$ : loading $(8 \mathrm{~min})$, waiting $(20 \mathrm{~min})$ and scanning (12 min). The load was continuously monitored by a strain gage (Honeywell 31E05KNO, USA). CT images were reconstructed using Nrecon (Skyscan, Belgium). A region of interested was defined as the entire scanning zone, which was limited to a $20 \mathrm{~mm}$ long cylinder in this pilot study (Fig. 1).

An algorithm was developed to automatically detect all beads from the reconstructed CT image slices and evaluate the micromotions of the beads attached to bone. This algorithm was divided in the following steps:

(1) Grayscale threshold to detect beads in CT slices.

(2) Bead center of mass computation using image grayscale values.

(3) Bead filtering and bead identification on all scans.

Using the initial unloaded case as a reference, the coordinate system of all other CT scans cases was rigidly transformed (Challis, 1995), so as to have the stem beads overlapping. The position of all bone beads of all scans was expressed in this local coordinate system. The coordinate system (Fig. 2, middle) was oriented such that the $x$-axis corresponded to the medio-lateral axis, the $y$-axis to the antero-posterior axis and the $z$-axis to the inferior-superior axis. Micromotion was defined as the difference between the actual (loaded) position and the reference (initially unloaded) position of the bone beads within the stem coordinate system. Mathematica (Wolfram Research, Inc., IL, USA) was used for image processing and analysis.

We choose to represent the micromotion error as the $95 \%$ prediction interval of the micromotion measurement. This interval was estimated as \pm 1.96 times the standard deviation of beads known to undergo zero micromotion. This zero-micromotion measurement was obtained from two datasets. The first one used stem beads at all loading steps of the previously mentioned experiment; this data was also used to evaluate the micromotion error dependency on load. The second one used bone beads from a second experiment. These beads were measured at different unloaded positions of the femur. In these two experiments, micromotion was obtained as the difference between the actual and the reference position of the beads.

\section{Results}

The error of micromotion measurement was evaluated at each of the 6 load amplitudes. The maximal error was less than $9.4 \mu \mathrm{m}$ in the axial $(z)$ directions and $5.2 \mu \mathrm{m}$ in the perpendicular directions $(x, y)$. Performing a Friedman's test for the dependency of the micromotion error on load gave $p$ values $>0.95$ for the null hypothesis that there is no load dependency on the $x, y$ and $z$ axis. Since there was no correlation between error and load, we used 

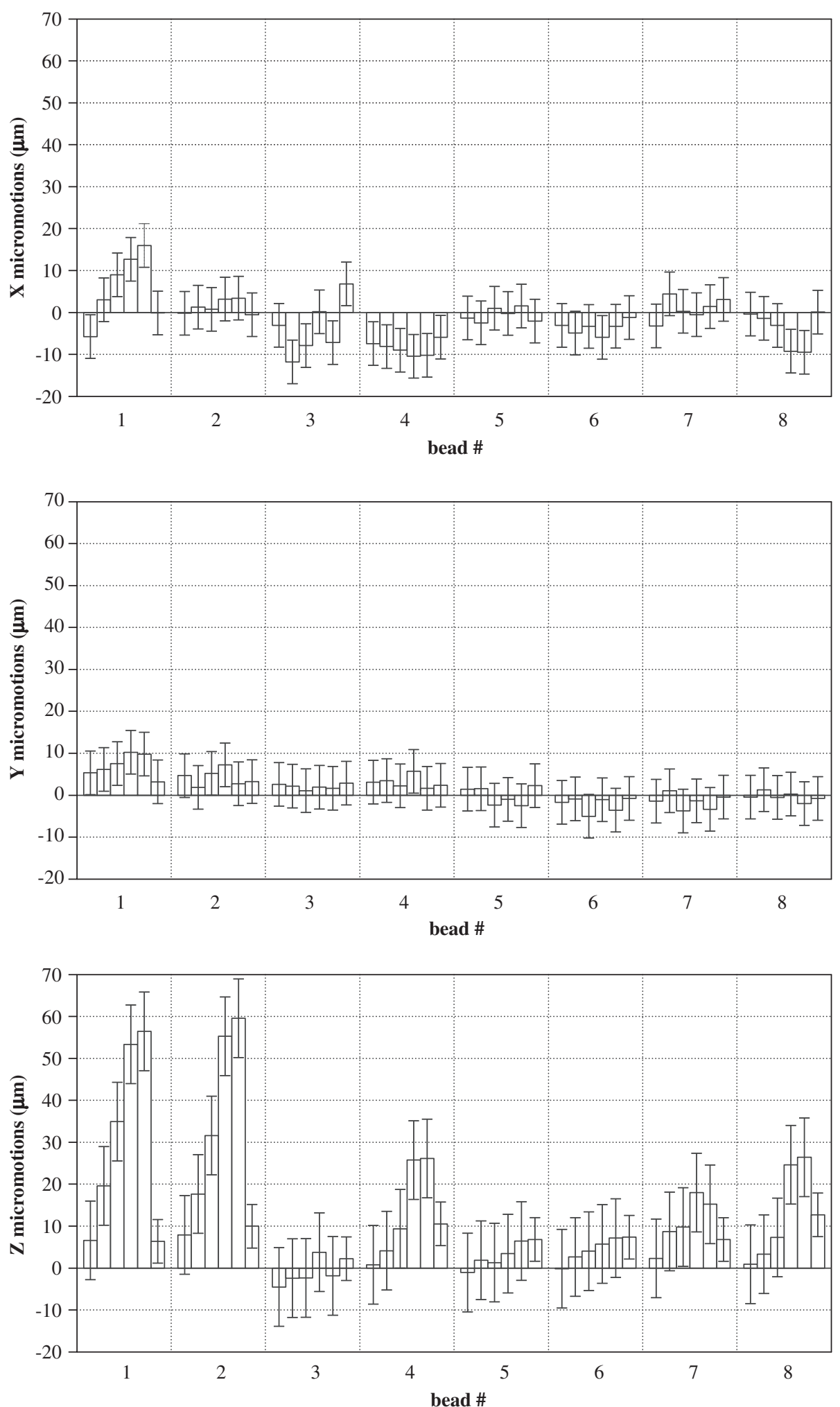

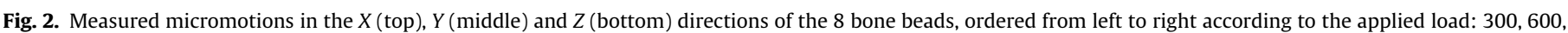
$900,1200,1400$ and back to $0 \mathrm{~N}$.

the maximal errors as the estimated error in the axial $(z)$ and perpendicular $(x, y)$ directions. The micromotion error measured using the second mentioned experiment was of $9 \mu \mathrm{m}$, which is consistent with the above result.

Within the region of interest, the detection algorithm identified all 8 beads present in this region. Micromotions were obtained for these 8 beads (Fig. 1), for each loading step. In the perpendicular direction $(x, y)$, micromotions showed a tendency to increase with load, but most variations remained within the error (Fig. 2). Axial ( $z$ ) micromotions increased with load, up to $60 \mu \mathrm{m}$ at $1400 \mathrm{~N}$. At maximal load, high micromotion was found in the superior anterior region (bead 1) and superior medial 
region (beads 2 and 4), and low micromotion was found in the lateral side (beads 3,5 and 6). When the stem returned to the unloaded state, the average micromotion was $8 \mu \mathrm{m}$ above the zero reference value of the initial unloaded state.

\section{Discussion}

Interfacial micromotions between bone and stem immediately after implantation are closely related to the failure of cementless total hip arthroplasty. Therefore, an experimental technique to measure the 3D map of interfacial micromotions would be an efficient tool to optimize stem design and surgical technique. This measurement is currently only possible in a limited number of simultaneous sites. The pilot study reported here has proved the feasibility to estimate locally the 3D interfacial micromotion at multiple and simultaneous points around the stem with an error lower than $10 \mu \mathrm{m}$. We have also verified that this error was sufficient to detect detrimental micromotions, and their location. The maximal amplitude of micromotion was consistent with typical reported values in the literature (Walker et al., 1987; Gilbert et al., 1992; Buhler et al., 1997; Britton et al., 2004).

Most of the experimental measurements of interfacial micromotion are using transducers (LVDT), but this technique provides in fact a measure of the displacement between the 2 fixation points, and not the local micromotions, which could overestimate the micromotions (Gheduzzi and Miles, 2007). In addition, this technique is rather difficult to setup, and allow only a limited number of simultaneous measurements in different locations. The strength of the proposed technique is to measure the local interfacial micromotions between the stem and the bone, for a large number of simultaneous locations around the stem, and with a rather simple and non-invasive method.

The developed technique has two main limitations: the maximal loading amplitude and the measurement error. The limitation in the maximal loading amplitude was only associated to the load cell, and can be easily extended by using another load cell. In this pilot study, the maximal compressive force corresponded to the plateau value (200\% of the bodyweight) during stance phase of the gait cycle (Bergmann et al., 2001). In the future, we might also add the abductor muscle forces. The reported maximal error corresponded to the maximal error in all directions and all loading cases. The error in one direction and one loading case was estimated as the standard deviation, among all stem beads, from the residues of the rigid body transformation. This error included all error sources: (1) scanning, (2) image processing, (3) non-rigid stem deformation and (4) metallic artifacts (which could also be considered as scanning error). Scanning and image processing parameters were optimized, but had slight influence on error. As discussed below, the stem deformation was also negligible. The most important source of error was then caused by the metallic artifacts, which could not be improved further by the scanning or image processing parameters (software correction was used). The overall maximal $95 \%$ error of the micromotion was of $9.4 \mu \mathrm{m}$ in the axial direction as well as in the perpendicular direction.

The maximal displacement between the stem beads was $0.7 \mu \mathrm{m}$, which support the rigid body assumption for the metallic stem. This displacement must be understood as the displacement of the beads caused by the non-rigid deformation of the stem only. This displacement was estimated for pure axial compression $(1400 \mathrm{~N})$ of a cylinder $(20 \mathrm{~mm}$ long with a cross sectional area of $330 \mathrm{~mm}^{2}$ ).
Since load was applied by a displacement controlled device, a decrease of load due to stress relaxation of bone was present during the scan. Waiting for $20 \mathrm{~min}$ between the load change and the scan was sufficient to prevent a load change of more than $1 \%$ during the $12 \mathrm{~min}$ of scan. We have further verified that the material used for the cylinder casing (AlMgSi1), which connects the bone anchoring point with the loading piston, induced a maximal displacement of the bone and stem of $0.09 \mu \mathrm{m}$ for every $10 \mathrm{~N}$ of load. Thus, $1 \%$ of load change was associated with approximately $0.1 \mu \mathrm{m}$ of displacement of the femur, which was fully acceptable for our measurements.

To conclude, we have evaluated the feasibility of an original technique to obtain local micromotions at the bone-stem interface. In a next step, this technique will be extended to get the quasi-continuous distribution of the micromotions around the stem. With this extension, the method could be used to validate finite element models, but also and most importantly, to compare and eventually improve the design of the cementless implants. The lifespan of these implants is indeed strongly dependent on the primary stability of the stem.

\section{Conflict of interest statement}

None of the authors has any conflict of interest.

\section{Acknowledgement}

This research was supported by a grant from the Swiss National Science Foundation (SNSF No. 125498) and by the Center of Translational Biomechanics EPFL-CHUV-DAL. The authors thank Symbios Orthopédie SA (Yverdon-Les-Bains, Switzerland) for donating implants and technical assistance.

\section{References}

Bergmann, G., Deuretzbacher, G., Heller, M., Graichen, F., Rohlmann, A., Strauss, J., Duda, G.N., 2001. Hip contact forces and gait patterns from routine activities. J. Biomech. 34 (7), 859-871.

Britton, J.R., Lyons, C.G., Prendergast, P.J., 2004. Measurement of the relative motion between an implant and bone under cyclic loading. Strain 40 (4), 193-202.

Buhler, D.W., Oxland, T.R., Nolte, L.P., 1997. Design and evaluation of a device for measuring three-dimensional micromotions of press-fit femoral stem prostheses. Med. Eng. Phys. 19 (2), 187-199.

Challis, J.H., 1995. A procedure for determining rigid body transformation parameters. J. Biomech. 28 (6), 733-737.

Cristofolini, L., Teutonico, A.S., Monti, L., Cappello, A., Toni, A., 2003. Comparative in vitro study on the long term performance of cemented hip stems: validation of a protocol to discriminate between "good" and "bad" designs. J. Biomech. 36 (11), 1603-1615.

Engh, C.A., O'Connor, D., Jasty, M., McGovern, T.F., Bobyn, J.D., Harris, W.H., 1992 Quantification of implant micromotion, strain shielding, and bone resorption with porous-coated anatomic medullary locking femoral prostheses. Clin. Orthop. Relat. Res. 285, 13-29.

Gheduzzi, S., Miles, A.W., 2007. A review of pre-clinical testing of femoral stem subsidence and comparison with clinical data. Proc. Inst. Mech. Eng. H 221 (1), 39-46.

Gilbert, J.L., Bloomfeld, R.S., Lautenschlager, E.P., Wixson, R.L., 1992. A computerbased biomechanical analysis of the three-dimensional motion of cementless hip prostheses. J. Biomech. 25 (4), 329-340.

Jasty, M., Bragdon, C., Burke, D., O’Connor, D., Lowenstein, J., Harris, W.H., 1997. In vivo skeletal responses to porous-surfaced implants subjected to small induced motions. J. Bone Joint Surg. Am. 79 (5), 707-714.

Pilliar, R.M., Lee, J.M., Maniatopoulos, C., 1986. Observations on the effect of movement on bone ingrowth into porous-surfaced implants. Clin. Orthop. Relat. Res. (208), 108-113.

Walker, P.S., Schneeweis, D., Murphy, S., Nelson, P., 1987. Strains and micromotions of press-fit femoral stem prostheses. J. Biomech. 20 (7), 693-702. 